\title{
The relationship between basic and applied research in universities
}

\author{
Peter James Bentley • Magnus Gulbrandsen • Svein Kyvik
}

Published online: 25 March 2015

(C) The Author(s) 2015. This article is published with open access at Springerlink.com

\begin{abstract}
What is the central research activity in modern universities? This paper uses a comprehensive survey among individuals from 15 countries to map differences in orientation towards basic/fundamental research, applied/practical research and a combination of the two. Despite some claims in the literature that basic research is no longer a preoccupation of universities, our findings point at a continued strong presence of basic research in universities but with large variations between countries and academic disciplines. At the individual level, most academics engage in a combination of basic and applied research, rather than specialising, with applied orientations generally more common. Academics specialising in basic research tend to receive less external funding, work in environments where applied research is less emphasised and hold weaker professional obligations to apply their knowledge to problems in society.
\end{abstract}

Keywords Basic research · Applied research · Mode 2 - Academic profession . Academic work

\section{Introduction}

The higher education literature is bursting with examples of how universities are changing in response to societal transformations and new demands. Many of these changes are

P. J. Bentley $(\bowtie)$

LH Martin Institute, The University of Melbourne, Level 1, 715 Swanston Street, Carlton, VIC 3010, Australia

e-mail: pbentley@unimelb.edu.au

M. Gulbrandsen

Centre for Technology, Innovation and Culture, University of Oslo, Postboks 1108, Blindern, 0317 Oslo, Norway

S. Kyvik

NIFU Nordic Institute for Studies in Innovation, Research and Education, P.O. Box 5183,

Majorstuen, 0302 Oslo, Norway 
described in rather pessimistic terms because they imply reduced funding, different activities and numerous tensions and challenges (Enders 2013). Only by protecting their socalled academic heartland can universities retain a degree of autonomy and a connection with widely shared values among their employees (Clark 1998). Long-term scientific work is at the centre of this heartland.

Basic research, or research undertaken with a primary purpose of the advancement of knowledge for its own sake, has traditionally been fundamental to university missions and their public funding. The "linear model" of innovation, popular during the post-1945 era, saw basic research as the primary contributor to innovation and economic growth, with universities as the institutional locus for public funding (Mowery and Sampat 2005). However, the centrality of basic research has come under question. The Mode 2 thesis, first outlined in The New Production of Knowledge by Gibbons et al. (1994), argued that disinterested, academic-oriented, disciplinary and autonomous research conducted exclusively within universities (Mode 1) is no longer the core mode of knowledge production. Mode 2 knowledge, produced within the context of application, has become the dominant form. Revisiting the Mode 2 discourse, Nowotny et al. (2003) summarised: “... the research that is variously described as 'pure', 'blue-skies', fundamental, or disinterested, is now a minority preoccupation-even in universities" (p. 184).

The Mode 2 diagnosis is broader than just the decline of fundamental research, and it also includes shifts towards collaborative and transdisciplinary research, greater heterogeneity in the sites of knowledge production, deeper social accountability and broader forms of quality control (beyond discipline-based peer review). The exceptionally wide scope of the Mode 2 discourse has been criticised for its lack of coherence and empirical validity (Hessels and Van Lente 2008). To date, there has been little evidence to support or refute Nowotny et al. (2003) claims, yet the Mode 2 discourse has influenced higher education and research policy makers and has been cited in almost ten thousand documents, with the vast majority of scientific papers treating it as an accepted or background concept (Hessels and Van Lente 2008).

Mode 2 is not necessarily new or unique, and it competes with other popular characterisations of the changing research agenda, some of which predate it. In the 1980s, Irvine and Martin (1984) identified the emergence of "strategic research", combining academic excellence with an orientation towards future practical utility. According to Rip (2004), strategic research is the basic way of carrying out research in the regime of "strategic science". However, strategic research retains a linearity between basic research and practical utility, including basic research as a cornerstone of its definition: "Strategic research [is] basic research carried out with the expectation that it will produce a broad base of knowledge likely to form the background to the solution of recognized current or future practical problems" (Irvine and Martin 1984). A similar conceptualisation, "translational research", shares many of these characteristics, focussing on the harnessing of basic research for practical uses, particularly within the health sciences (Woolf 2008). Other conceptualisations, such as "finalised science" and "post-normal science", also emphasise strong practice orientation, but these concepts are much more nuanced in their interpretation, limiting their implications to certain disciplines or policy-relevant fields (Weingart 1997).

More recently, the triple helix model of university-industry-government relations has also had considerable impact on research policy (Etzkowitz and Leydesdorff 2000). Like Mode 2, it contends that the future legitimation of science depends upon application orientation and contribution to economic development. However, it distinguishes itself from Mode 2 because universities retain distinctiveness as the core knowledge institutions and the application orientation process is emergent, rather than complete (Etzkowitz and 
Leydesdorff 2000). Academic capitalism also stresses the growing engagement of academics in application-oriented and commercially funded research, but the extent of such engagement is limited based on the closeness of one's discipline to the market (Slaughter and Leslie 1997). Overall, the Mode 2 claims regarding the shifting research agenda away from fundamental research is unique only in its comprehensiveness, particularly with reference to country and disciplinary context.

This study is in particular interested in the claim that universities are no longer preoccupied with basic or theoretical research. Our decision to limit ourselves to this component follows Hessels and van Lente's (2008) recommendation that Mode 2's impact necessitates empirical research on each of the constitutive parts separately. We do so by drawing on data from an international survey among academic staff in research universities in 15 countries and across 10 academic fields. The lack of international comparable indicators has been a crucial limitation for understanding how universities operate in national innovation systems (Mowery and Sampat 2005, p. 234). The comparative dimension allows us to test whether the assumptions surrounding Mode 2 apply to different contexts. Firstly, we investigate the extent to which individual academics worldwide consider basic or theoretical research a core part of their current research. Secondly, we examine the extent to which academics specialise only in basic or applied research, or combine these activities. Thirdly, we investigate variations in the extent to which academics specialise only in basic or applied research. Why might some academics within certain countries and academic fields choose to engage in both types of research, while others choose not to? We investigate three possible explanations. We expect engagement in basic and applied research to relate to: access to external commercial research funding; institutional expectations/policies; and individual normative/social behaviour.

Question 1: To what extent is basic research still a defining characteristic of universities across the world?

Nowotny et al. (2003) are clear regarding three generalisable causes for the decline in fundamental research: increased steering of research priorities; increased commercialisation of research (resulting from decreased public funding and increased attention to intellectual property ownership); and broader accountability of science.

The limited evidence of how individual academics view their primary research points to the contrary that basic research continues to be sustained as a major activity. A large-scale Finnish investigation found that an "academic orientation" with strong emphasis on basic research remains central across disciplinary fields (Ylijoki et al. 2011). The authors question the thesis that a significant transformation is taking place in universities, arguing instead that academics find different ways to combine an academic orientation with various forms of societal engagement. A UK interview study found the same, although it also emphasised how the term "basic research" is flexible and may be adapted to varying circumstances (Calvert 2006). Gulbrandsen and Kyvik (2010) also found that $90 \%$ of Norwegian academics undertook basic research, with slightly less than half declaring their research "very much" basic (maximum value on a five-point scale), confirming other findings that basic research remains a strong identity marker for university staff (Gulbrandsen and Langfeldt 2004). These results are indicative of the resilience of basic research within universities, but may not be generalisable to other countries. Public funding of university research retains a strong position in Norway (Kyvik 2007). 
National context is potentially of greater importance to understanding changes in knowledge production than implied by the Mode 2 thesis. Even if one acknowledges that universities across the world have experienced increased steering of research priorities and accountability pressures, it is unlikely that the effects would be uniform across countries. Shinn (2002) argues there is little evidence that science has become de-nationalised and it is inappropriate to generalise broad changes across national settings. According to Shinn, despite globalisation trends, universities, business and government still function predominantly within the national settings. The interaction between these institutions has implications for university governance and research behaviours. European countries have stronger traditions of academic self-determination, whereas market coordination has stronger traditions in most English-speaking countries and state control in parts of Asia (Clark 1983). Even within regions of similar academic traditions, convergences in higher education policies have not diluted the national character of academic labour markets and their internal regulation of research (Musselin 2005). This makes a data set from different countries particularly valuable.

National governments remain the main funder of university research in almost all OECD countries, but this varies from over $80 \%$ of total funding of $\mathrm{R} \& \mathrm{D}$ in higher education institutions in Argentina, Australia, Italy, Finland, Germany, Norway and the Netherlands to closer to two-thirds in the USA, UK and Canada, and just over half of all funding in China (OECD 2013). Countries also differ in how this public funding is allocated to universities for research. According to Auranen and Nieminen (2010), core public research funding is determined predominantly via input-orientated measures in many European countries (e.g. Norway, Finland, Netherlands and Germany), whereas output-oriented funding models are more dominant in Australia and the UK. Although many European countries have been moving towards output-oriented funding models, there has been stronger resistance from universities and their implementation has been weaker. This likely reflects the traditionally stronger academic oligarchy within these countries (Clark 1983).

The implications for engagement in basic research is not directly clear, but one would reasonably assume that country-level differences in how universities are governed and funded would lead to greater diversity in research behaviours. For example, systems with greater levels of core funding based on input measures have higher levels of stability compared to output-oriented and externally funded systems (Auranen and Nieminen 2010). By implication, risky, fundamental and disinterested basic research may be more likely to flourish in the countries with more predictable funding. Although we cannot test empirically why country-level differences in basic research engagement may exist (countries differ across a range of idiosyncratic characteristics, not just governance or funding), it is appropriate to test the robustness of Nowotny and colleague's general claims.

Question 2: To what extent do academics specialise only in basic or applied research, or combine these activities?

If universities are no longer preoccupied with pure, fundamental and disinterested research, what have become the dominant research preoccupations? Nowotny et al. (2003) discuss how Mode 2 knowledge production is characterised by diversity, reflexivity and a shift away from the stable, clear and unchallengeable taxonomy of disciplinary research. Further, the original Mode 2 argument stated that Mode 2 did not replace Mode 1; it supplemented and grew alongside it (Gibbons et al. 1994). Accordingly, individual academics engaged in Mode 2 knowledge production may continue to be involved in basic research alongside applicationoriented research. Adding further complexity, application orientation (and the four other 
attributes characterising Mode 2 knowledge) is "not present in every instance of Mode 2" (Gibbons et al. 1994, p. 8). Thus, application orientation is neither a prerequisite, nor a sufficient, characterisation of Mode 2. This is similar to Stokes' (1997) argument that considerations of use often go hand in hand with a quest for fundamental understanding. Despite these challenges, one may reasonably understand Nowotny et al. (2003) argument to imply a shift in the balance between basic and applied research, towards more applied research specialists.

The few studies that have reported on this issue indicate, however, that a traditional academic orientation is resilient rather than new combinations. Albert (2003) found no evidence that Canadian academics in sociology and economics departments had shifted their research attention away from disciplinary research intended for their peers and begun specialising in interdisciplinary and practical research. Studies of scientists in the Netherlands (van Rijnsoever and Hessels 2011) and Finnish academics in technology, social science and humanities departments (Hakala and Ylijoki 2001) also indicate high commitment to Mode 1-type research over purely applied research. Finally, Gulbrandsen and Kyvik (2010) found that few Norwegian academics in 2000 were engaged exclusively in basic research, but this was still greater than the proportion engaged exclusively in applied research, although a combination was most common for all university staff members. Each of these aforementioned studies is somewhat limited by their national context. Our international data provide us with a useful opportunity to examine this relationship.

Another contextual limitation of the Mode 2 thesis is academic field (Hessels and Van Lente 2008). Disciplinary cultures span international boundaries and influence what types of research are considered legitimate within the scientific community (Becher 1994). Weingart (1997) argues that Mode 2 attributes, such as application-oriented research, make little sense in scientific disciplines distanced from policy makers. Likewise, Godin (1998) notes the difficulty certain disciplines face when modifying their research towards applied and practical purposes. According to Becher (1994), academic disciplines differ in their cognitive knowledge structure from pure (theoretical) to applied (pragmatic). Applied disciplines within the technological sciences (e.g. engineering) are purposive and pragmatic in their knowledge, oriented towards mastery of the physical environment, resulting in products/techniques. In the pure sciences (e.g. physics), knowledge is cumulative, atomistic, well organised and results in discovery or explanation, rather than directly practical outputs. Social sciences and humanities may also be pure or applied, depending on whether they are oriented towards practical, utilitarian and functional improvements to professional practice (e.g. education), or understanding and interpretation (e.g. humanities).

Based on Becher's typology, we may expect specialisation in basic research to be more common in the hard-pure disciplines (e.g. life sciences and physical sciences) and soft-pure disciplines (humanities and social sciences). By contrast, specialisation in applied research may be more common in the science-based professions within hard-applied disciplines (e.g. agriculture, engineering, medical sciences) and social professions within the soft-applied disciplines (e.g. education, law). Our data will allow us to extend disciplinary-based findings from specific countries to an international context, controlling for other individual-level factors.

Question 3: What might explain individual differences in specialisation versus combining basic and applied research?

Few universities are completely insulated from the increased steering of research priorities, commercialisation and accountability pressures, but this does not imply that individual academics will respond in consistent and uniform ways. Despite the decline in autonomy, 
academics still retain a relatively high degree of discretion over their non-teaching hours and have the freedom to seek research activities of professional and personal engagement, although the latter may not always be compatible with a secure academic career (Davies 2013). Why might some academics in comparable disciplines and countries engage predominantly in basic research, while others do not? We put forward three possible explanations: commercial research funding, institutional expectations and professional norms.

Nowotny et al. (2003) argue that inadequacy of public funding has caused universities to shift their research preoccupation away from basic research. Government core funding of research has declined in a range of OECD countries, and the mechanisms for disbursing public funds have shifted from stable block grants towards results-based models

(Auranen and Nieminen 2010). Given that basic research leads to unpredictable results and tends to attract little private sector funding, we may expect that access to business and industry funding will also be a differentiator between individual academics for basic and applied research engagement within countries. Academics able to source business and industry funding for research are less reliant on dwindling institutional funding and may be more likely to be engaged in applied research.

A second important factor is institutional expectations for research. Universities across the world have seen stakeholder expectations widen and deepen, including the expectations for producing practical knowledge and training. Clark (1998) describes the situation in terms of a demand-response imbalance: "demands on universities outrun their capacity to respond" (p. 6). In terms of functional differentiation, modern universities are characterised by "overcomplexity and underdifferentiation" (Enders 2013). However, universities are not entirely victims of past history and stakeholder demands. Universities are capable of choosing which stakeholder demands to respond to, where within the organisation these responses will occur and how. For example, entrepreneurial responses may occur at the "developmental periphery", where traditional disciplinary research meets the outside world through interdisciplinary research, contract research, contract education and consultancy (Clark 1998). Such engagement with external stakeholders can diversify the funding base, promote an entrepreneurial culture throughout the university, as well as stimulate the "academic heartland" where traditional disciplinary and theoretical research is maintained. In other words, universities have the capability to respond to stakeholder needs by promoting commercial and interdisciplinary research. Therefore, we may expect that academics who work in such environments will be more likely to engage in applied research and not necessarily to the detriment of their engagement in basic research.

A third factor explaining diversity is professional norms. One of the defining characteristics of academia is autonomy and protection from external influence. The freedom of professors to teach without external control was a core principle of the mediaeval university and has influenced the development of universities, not just in Central and Western Europe, but also in the USA (Altbach 2001). The emergence of privately funded, practically oriented research for commercial or technology transfer purposes in the mid- to late twentieth century challenged established scientific norms, particularly the norms of disinterested research and public ownership of knowledge (Stuart and Ding 2006). The changing funding patterns have been described as placing the future of basic research in jeopardy (Altbach 2001). However, scientific norms are not static. Etzkowitz (1998) argues that scientific norms have progressively accepted commercial research and academic entrepreneurialism with profit-making motives. These normative changes may be considered the "second revolution" in academia, following the first revolution which made research a core function of universities. Given the challenges that applied research poses to traditional scientific norms, we expect that engagement in applied research will reflect how 
individuals view applied research. Those who view applied research as a professional obligation will be more likely to be more engaged in applied research.

\section{Data and methodology}

The data come from the changing academic profession (CAP) international survey of the academic profession across 19 countries during 2007-2008: Argentina, Australia, Brazil, Canada, China and Hong Kong, Finland, Germany, Italy, Japan, Malaysia, Mexico, the Netherlands, Norway, Portugal, South Africa, Republic of Korea, UK and the USA. The CAP project used a common random sampling protocol and survey instrument. The target population of the CAP survey was professionals in universities or other higher education institutions offering a baccalaureate degree or higher. Given that this article addresses research orientation, we restrict ourselves to academics employed in universities with research and teaching missions, generally those universities awarding doctorate degrees (i.e. excluding polytechnics, teaching-focused colleges, research institutes). We exclude four of the 19 countries from our analysis. This is due to an inability to differentiate between teachingfocused and research-focused universities or small sample sizes (Japan and Korea), low response rate (Portugal) or limited information on the sampling framework (South Africa).

The response rates, including partially completed responses, were mostly below 40 per cent. Therefore, there is a potential risk of non-response bias in some countries. However, national samples were found broadly representative of the respective populations on strata such as gender, academic rank and institutional type (Cummings and Finkelstein 2012; RIHE 2008; Vabø and Ramberg 2009). Further, there are few reasons to expect that nonrespondents would differ substantially in terms of self-declared research orientation. After removing non-respondents to the core questions regarding basic and applied research orientation and respondents not employed in academic ranks, we were left with a sample of 12,379 academics (Table 1).

Table 1 Response rates and sample sizes by country

\begin{tabular}{llrr}
\hline & Response rate $(\%)$ & Total survey respondents & Valid cases $(n)$ \\
\hline Argentina & 34 & 826 & 760 \\
Australia & 25 & 1,370 & 934 \\
Brazil & 26 & 1,147 & 403 \\
Canada & 17 & 1,152 & 927 \\
China & 86 & 3,612 & 2,459 \\
Finland & 29 & 1,452 & 464 \\
Germany & 32 & 1,265 & 840 \\
Hong Kong & 13 & 811 & 644 \\
Italy & 35 & 1,701 & 1,296 \\
Malaysia & 30 & 1,220 & 729 \\
Mexico & 69 & 1,973 & 530 \\
Netherlands & 18 & 1,167 & 447 \\
Norway & 36 & 1,035 & 529 \\
UK & 15 & 1,565 & 716 \\
USA & 23 & 1,146 & 701 \\
Total & 28 & 21,442 & 12,379 \\
\hline
\end{tabular}


Dependent variable: measuring basic and applied research

We consider Nowotny et al. (2003) references to "pure", "blue skies", "fundamental" or "disinterested" research to be analogous to basic or theoretical research and context of application to be analogous to applied or practically oriented research. Our data for measuring research orientation come directly from the CAP survey. The relevant question asked: "How would you characterize the emphasis of your primary research this (or for the previous) academic year?" Respondents provided a score ranging from "very much" (1) to "not at all" (5) on seven separate criteria. This study examines the results for two of these criteria: "basic/theoretical" and "applied/practically oriented". The explicit reference to theory and practical orientation is consistent with the extended definitions of basic and applied research in the OECD's Frascati Manual (2002). Given that respondents provided scores on each criterion separately, it was up to individual to determine whether the concepts of basic and applied research were mutually exclusive or inclusive.

We are interested both in the extent to which basic research is a defining characteristic of academic work, as well as its relationship with applied research. We distinguish between five distinct research orientations: pure basic; leaning towards basic; equally, basic and applied; leaning towards applied; and pure applied. A sixth category for respondents with neither basic nor applied research orientation is a residual category for those not declaring a clear research orientation. The definition of each research orientation group and number of respondents in each group is presented as a cross-tabulated matrix in Table 2.

There are certain limitations which may affect how the research results are interpreted. Firstly, there is a risk that the concepts of "basic", "theoretical", "applied" and "practically oriented" may be understood differently across countries, particularly after translation into local language. In countries where English was not the language of the survey, most used a combination of professional translators, academic translators, survey piloting and peer review (e.g. regions with common languages), in addition to internal review by the project team.

Although we are confident the concepts of basic and applied research were translated accurately, there is a risk the concepts are interpreted differently, particularly in countries where research has not traditionally been conducted within universities. Secondly, one assumes that differences in response to Likert-type questions reflect differences in substantive engagement and that engagement is related to contextual factors such as research norms of the discipline or institution. However, academics may exaggerate or define their research in accordance with norms, rather than contextual factors influencing substantive engagement.

Independent variables: factors associated with research orientation

The operationalisation of independent variables is outlined below. Means on each variable are listed for each country in Table 4 of "Appendix".

Country is a dichotomous variable based on country of employment: Argentina; Australia; Brazil; Canada; China; Finland; Germany; Hong Kong; Italy; Malaysia; Mexico; Netherlands; Norway; UK; and USA.

Academic discipline is a dichotomous variable based on discipline of one's current academic unit: education (teacher training and education science); humanities (humanities and arts); social sciences (social and behavioural sciences); commerce (business and administration, economics); law; life sciences; physical sciences (physical sciences, mathematics, computer sciences); engineering (engineering, manufacturing and construction, architecture); agriculture; medicine (medical sciences, health-related sciences, social services); and other (personal services, transport services, security services or other). Based on Becher's (1994) 
Table 2 Research orientation categorisation and definition, based on: "How would you characterize the emphasis of your primary research this (or the previous) academic year?"

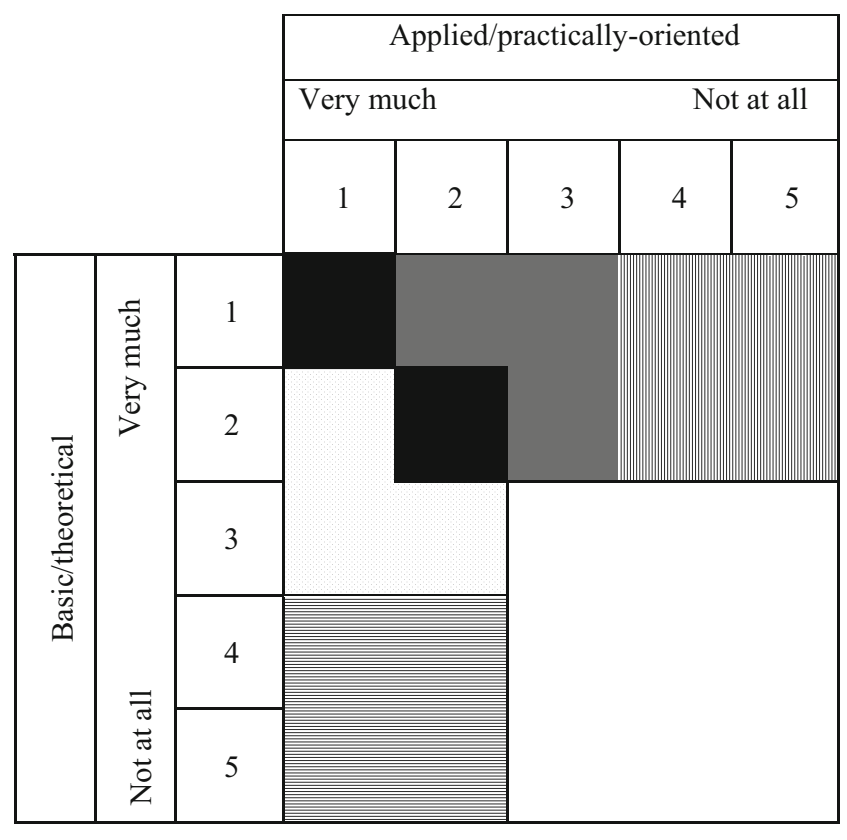

\section{Category}

Equally, basic and applied:

Leaning towards basic:

Leaning towards applied

Pure basic

Pure applied

Neither basic nor applied

\section{Definition}

Very much (1) or high (2) for both basic and applied (3,133 respondents).

Very much (1) or high (2) for basic, and to a lesser extent ( 2 or 3 ) for applied (2,048 respondents).

Very much (1) or high (2) for applied, and to a lesser extent ( 2 or 3$)$ for basic (2,722 respondents).

Very much (1) or high (2) for basic and little (4) or Not at all (5) for applied (1,547 respondents).

Very much (1) or high (2) for applied, and little (4) or Not at all (5) for basic (1,931 respondents).

(3) or less for both (998 respondents).

disciplinary typology, we expect basic research to continue to be a core preoccupation of academics within hard-pure sciences and soft-pure social sciences and humanities.

Commercial research funding is the percentage of one's research funding (in the current or previous academic year) which came from business firms or industry. We expect that engagement in applied research will be greater among those academics with greater access to external commercial research funding.

Individual normative/social behaviour includes one scale variable based on the ordinal responses (from "strongly agree" [5] to "strongly disagree" [1]) to the following statement: "Faculty in my discipline have a professional obligation to apply their knowledge to 
problems in society". We expect that engagement in applied research will reflect the extent to which individuals view applied research as a professional obligation.

Institutional norms and expectations/policy/strategy includes one scale variable based on the ordinal responses (from "strongly agree" [5] to "strongly disagree" [1]) to the following statement: "Your institution emphasizes commercially-oriented or applied research". We expect that academics working in environments emphasising commercially oriented and applied research will tend to engage more in applied research.

\section{Results}

Basic research as a pre-occupation

Our first research question addressed the extent to which basic research is still a defining characteristic of universities. When asked to characterise the emphasis of their primary research in the previous year on a scale of 1 ("very much") to 5 ("not at all"), $61 \%$ reported significant engagement in basic research (values of 1 or 2). Very few academics (7\%) reported being "not at all" engaged in basic research, with the remainder engaged partly in basic research (32\%). Based on these results, Nowotny et al. (2003) claim that fundamental research is a minority preoccupation in universities received little empirical support, with $93 \%$ reporting at least some engagement with basic research. At the very least, claims that fundamental research is a minority preoccupation should be qualified by country. Levels of significant engagement in basic research ranged from $78 \%$ in China to half of all academics in Argentina (50\%). Universities in Argentina, and to a lesser extent, Brazil, tend to employ a large number of part-time and teaching-only staff, but this does not account for their relatively weak engagement in basic research. Part-time staff in these countries did not differ from their full-time colleagues in their engagement in basic research.

Although most academics engage in basic research, a greater proportion report engagement in applied research. To this extent, fundamental research may not be the primary preoccupation of universities, even though the majority engage in it. More than two-thirds (69\%) of academics characterised their research as applied to a large extent, one quarter (25\%) were partly engaged in applied research, and $6 \%$ not at all. China was again the country where academics were most likely to report strong engagement in applied research $(86 \%)$. Norway and the Netherlands were the only countries where more academics reported significant engagement in basic research compared to applied research. The proportion of academics in each country reporting significant engagement in basic research (values of 1 or 2 ) is shown in Fig. 1 on the left-hand bar, while the proportion reporting significant engagement in applied research is shown on the right-hand bar. Note that the basic and applied characterisations were derived from separate questions. Therefore, it was up to the respondent to decide whether the two categories (basic and applied) were mutually exclusive characterisations. The fact that sum of both bars exceeds $100 \%$ in all countries is evidence that many academics do not consider these characterisations as mutually exclusive.

Specialisation versus the coexistence of basic and applied research

In order to investigate the relationship between basic and applied research, and tendency towards specialisation, we exclude respondents who were not sufficiently engaged in basic or applied research. Rather than being engaged in research outside the basic/applied typologies, academics with no clear research orientation are better understood as insufficiently research active. For 


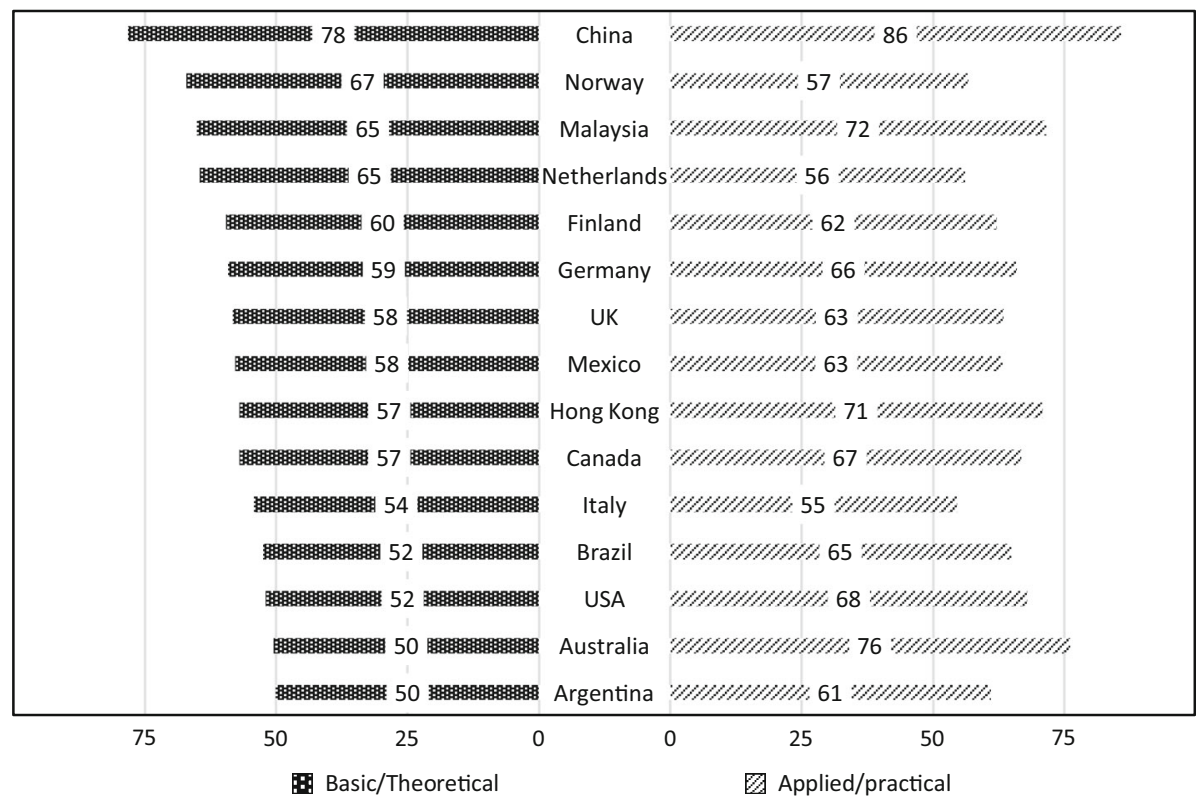

Fig. 1 Proportion of academics whose primary research was to a considerable extent basic/theoretical (left-hand bar) and applied/practical (right-hand bar), by country

example, they published little, spent very few hours on research and were also unlikely to characterise their research in other ways (e.g. commercially oriented, single disciplinary or interdisciplinary). These 998 respondents were classified as "neither basic nor applied" in Table 2 ( $8 \%$ of the total sample) and are subsequently excluded from the valid cases for further analysis.

Among academics with clear research orientations, $14 \%$ specialised as "pure basic" researchers. On this basis, only a small minority of academics may be understood to be preoccupied entirely with basic research. However, the lack of specialisation was not primarily due to a tendency to specialise in applied research, and only $17 \%$ could be considered "pure applied" researchers. The bulk of the sample engaged in both basic and applied research. The most frequent categorisation was "equally, basic and applied" (27\%), followed by "leaning towards applied" (24\%) and "leaning towards basic" $(18 \%)$. In other words, basic research is not the primary preoccupation of most academics, but this is because it is integrated with applied research in most cases.

Specialisation varied considerably across countries. Pure basic researchers were most common in Italy, Netherlands and Norway, comprising roughly one quarter of all academics and outnumbering those in the pure-applied category. At the other extreme, almost no academics were specialised in basic research in China (2\%) and Malaysia (5\%). However, this reflected a greater tendency to combine basic and applied research in these countries, rather than an aversion to basic research. The balance between basic and applied research was most skewed in Australia, where pure applied researchers were more than twice as common as pure basic researchers. The proportion of academics specialising in basic or applied research by country is shown in Fig. 2. The full breakdown across the five research orientation categories is shown for each country in Table 5 of "Appendix".

Research specialisation was closely aligned with Becher's (1994) disciplinary typology of pure/theoretical and applied/pragmatic knowledge structures. Pure basic researchers were most 


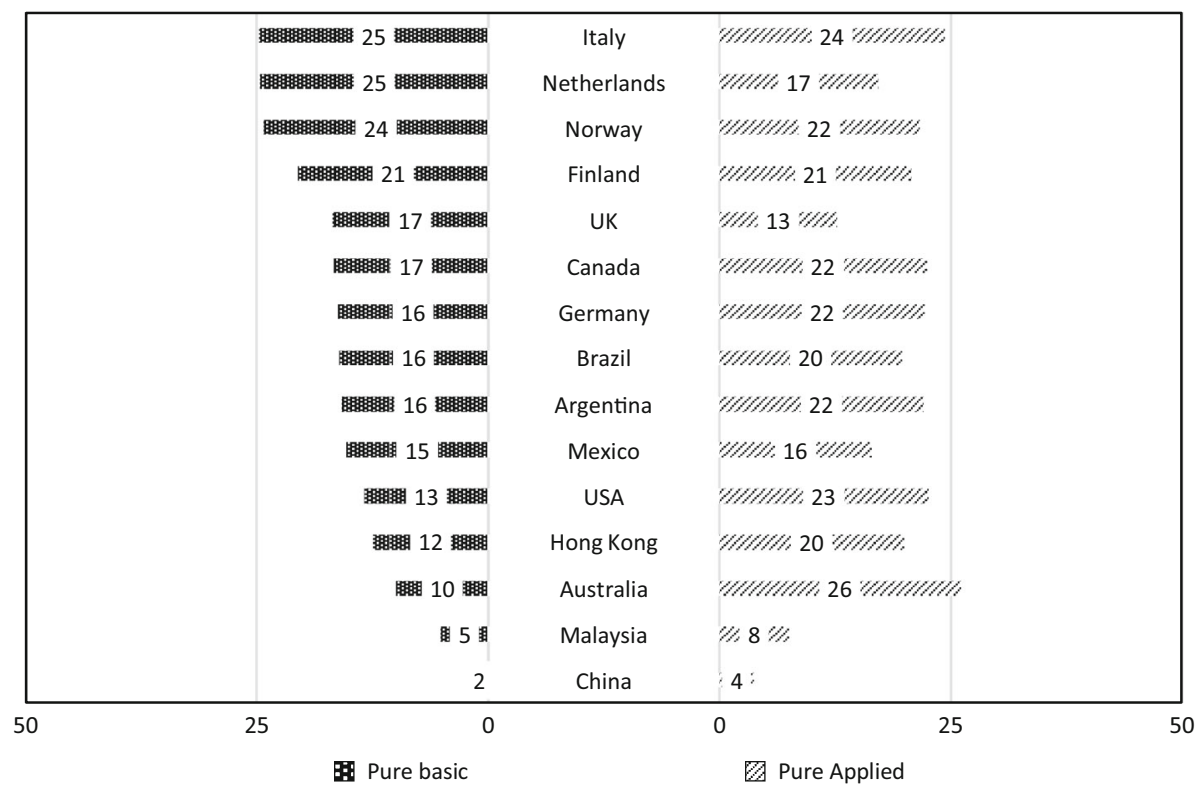

Fig. 2 Proportion of academics engaged purely in basic/theoretical research (left-hand bar) and purely applied/practical research (right-hand bar), by country

common in the humanities (28\%), life sciences (22\%), social sciences (19\%) and physical sciences $(18 \%)$. These discipline-based differences were broadly consistent across country samples, with humanities ranked in the top 3 basic research disciplines in all countries other than Australia. Comparably, few pure basic researchers were located in Becher's applied disciplines within the science-based and social professions, such as medicine (9\%), business studies $(8 \%)$, education (7\%), agriculture (4\%) and engineering (3\%). The balance between specialisation in basic or applied research also aligns with Becher's typology, with few disciplines having a roughly equal number of pure applied and pure basic researchers. One possible exception may be law, where pure applied research was rare (10\%) compared to pure basic $(18 \%)$. This appears to contradict the practice orientation of the discipline. However, it may be that purely "practically oriented" research is defined differently by law academics, given that research within a legal practice involves direct remuneration for specific clients. The proportion of academics specialising in basic or applied research by discipline is shown in Fig. 3 (full breakdown by discipline in Table 6 of "Appendix").

Compared to the country-level results where pure applied researchers were only marginally more common than pure basic researchers, research orientation is considerably more fragmented across disciplines. This also supports Becher's claims that disciplinary cultures often span national boundaries and embody collective norms. Taken together, the large differences across countries and disciplines suggest that claims regarding basic, theoretical or blue sky research should be nuanced with reference to national and disciplinary context.

\section{Multinomial results}

Our third research question was how we might account for individual-level differences in engagement and specialisation in basic and applied research. From the above results, the disciplinary implications are clear. Given that countries differ in their proportion of 


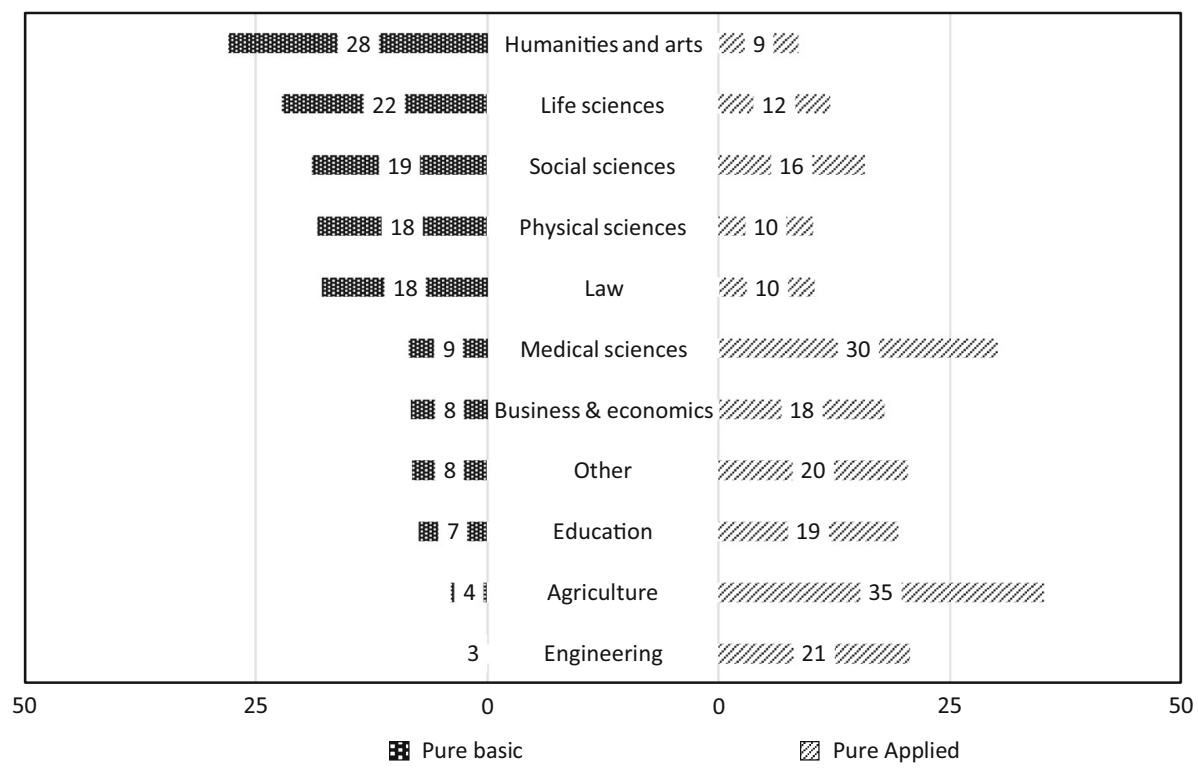

Fig. 3 Proportion of academics engaged purely in basic/theoretical research (left-hand bar) and purely applied/practical research (right-hand bar), by discipline of current academic unit

academics by discipline, cross-country comparisons need to control for these differences. However, significant variation within countries and disciplines suggests that other factors may also influence specialisation. To examine this further, we computed a multinomial logistic regression with three categorical outcomes: pure basic, pure applied and basic and applied as the reference category (merging the three categories which involved both types of research). Controlling for country and discipline, we investigated whether specialisation in basic or applied research was also associated with differences in access to commercial research funding, institutional expectations/policies and individual normative/social behaviour. We also checked whether the results differed when controlling for demographic characteristics (gender, age and age squared), but the main results did not change. The multinomial logistic regression results are presented in Table 3.

The likelihood ratio tests from the multinomial regression results (not shown) indicated a statistically significant relationship between research specialisation and commercial research funding, organisational expectations and individual norms. However, organisational expectations for commercially oriented or applied research (institutional norms) were not a statistically significant factor differentiating between all categories of research specialisation. Compared to the reference category for those engaged in both basic and applied research, and holding other factors constant, a one unit increase in agreement (on a five-point scale) with the statement "Your institution emphasizes commercially-oriented or applied research" was only statistically significant in its negative association with being a pure basic researcher (with a decrease in the multinomial log odds of 0.15 per unit increase). Agreement with this statement was not significant in its relationship with being a pure applied researcher. In other words, institutional norms emphasising research commercialisation appear to increase engagement in applied research but not specialisation in this type of research. On the other hand, individual norms were significantly associated with both types of specialisation. A one unit increase in agreement with the statement that "Faculty in my discipline have a professional 
Table 3 Multinomial logistic regression results, factors associated with pure basic and pure applied research orientations

\begin{tabular}{|c|c|c|c|c|c|c|}
\hline & \multicolumn{3}{|c|}{ Research orientation $=$ pure basic } & \multicolumn{3}{|c|}{ Research orientation $=$ pure applied } \\
\hline & $B$ & SE & $\operatorname{Exp}(B)$ & $B$ & SE & $\operatorname{Exp}(B)$ \\
\hline Intercept & $0.63 * *$ & 0.21 & & $-2.12 * *$ & 0.20 & \\
\hline Education & $-0.66 * *$ & 0.20 & 0.52 & 0.26 & 0.15 & 1.30 \\
\hline Humanities & $0.50 * *$ & 0.12 & 1.65 & $-0.43 * *$ & 0.15 & 0.65 \\
\hline Commerce & $-0.51 * *$ & 0.16 & 0.60 & $0.36 * *$ & 0.14 & 1.43 \\
\hline Law & 0.18 & 0.21 & 1.20 & -0.33 & 0.25 & 0.72 \\
\hline Life sciences & 0.05 & 0.14 & 1.06 & -0.25 & 0.16 & 0.78 \\
\hline Physical sciences & -0.09 & 0.12 & 0.91 & -0.22 & 0.13 & 0.80 \\
\hline Engineering & $-1.27 * *$ & 0.18 & 0.28 & $0.48 * *$ & 0.12 & 1.62 \\
\hline Agriculture & $-1.57 * *$ & 0.35 & 0.21 & $0.78 * *$ & 0.16 & 2.19 \\
\hline Medicine & $-0.66 * *$ & 0.15 & 0.52 & $0.67 * *$ & 0.12 & 1.95 \\
\hline Other & -0.36 & 0.24 & 0.70 & 0.36 & 0.19 & 1.44 \\
\hline ARG & 0.20 & 0.19 & 1.22 & 0.09 & 0.15 & 1.10 \\
\hline AUS & 0.00 & 0.20 & 1.00 & 0.27 & 0.15 & 1.31 \\
\hline BRA & 0.13 & 0.26 & 1.14 & 0.05 & 0.22 & 1.05 \\
\hline CAN & 0.25 & 0.18 & 1.29 & 0.08 & 0.15 & 1.08 \\
\hline $\mathrm{CHI}$ & $-1.92 * *$ & 0.24 & 0.15 & $-2.22 * *$ & 0.18 & 0.11 \\
\hline FIN & $0.65 * *$ & 0.21 & 1.92 & 0.09 & 0.19 & 1.10 \\
\hline GER & 0.22 & 0.18 & 1.24 & 0.06 & 0.15 & 1.06 \\
\hline HK & -0.07 & 0.21 & 0.93 & -0.10 & 0.16 & 0.90 \\
\hline ITA & $1.04 * *$ & 0.17 & 2.83 & $0.38 * *$ & 0.14 & 1.46 \\
\hline MAL & $-0.78 * *$ & 0.27 & 0.46 & $-1.24 * *$ & 0.21 & 0.29 \\
\hline MEX & 0.20 & 0.25 & 1.22 & -0.36 & 0.22 & 0.70 \\
\hline NET & $0.54 * *$ & 0.20 & 1.72 & 0.01 & 0.19 & 1.01 \\
\hline NOR & $0.70 * *$ & 0.19 & 2.02 & 0.23 & 0.17 & 1.26 \\
\hline UK & 0.05 & 0.19 & 1.05 & $-0.61 * *$ & 0.18 & 0.54 \\
\hline Commercial res. funding & $-0.03 * *$ & 0.00 & 0.97 & $0.01 * *$ & 0.00 & 1.01 \\
\hline Institutional norms & $-0.15^{* *}$ & 0.03 & 0.86 & -0.01 & 0.03 & 0.99 \\
\hline Individual norms & $-0.46^{* *}$ & 0.03 & 0.63 & $0.20 * *$ & 0.03 & 1.22 \\
\hline
\end{tabular}

$N=8654$

Model fit: $-2 \log$ likelihood (final) 8,277.046; Chi-square 2,253.616; $d f$ 54; sig. 0.000

Pseudo R-Square: Cox and Snell 0.229; Nagelkerke 0.281; McFadden 0.154

Reference categories: both basic and applied (dependent variable); USA; social sciences

Significance level: $* * p<0.01 ; * p<0.05$

obligation to apply their knowledge to problems in society" was associated with an increased likelihood of being pure applied. Agreement was even more strongly associated with a decrease in the likelihood of being a pure basic researcher.

External commercial research funding was a significant differentiator for research specialisation, but mostly in its negative relationship with pure basic research specialisation. A one point percentage increase in the proportion of one's research funding coming from business or government sources was associated with a small but statistically significant decrease in the likelihood of being a pure basic researcher. To a lesser extent, 
commercial research funding was also positively associated with pure applied research. In other words, commercial research funding tends to increase the likelihood of being engaged in applied research, but mostly in combination with basic research rather than in specialisation. This relatively minor relationship is partly due to the small share of academics for whom commercial funding is a main source of research funding. In most countries, the mean proportion of research funding from business and industry was around $5 \%$ or less. However, in Germany and China, these proportions were considerably greater at 10 and $15 \%$, respectively (see Table 4 of "Appendix"). This is consistent with their high proportion of higher education R\&D expenditure financed by industry, at 14 and $33 \%$, respectively, versus $6 \%$ across all OECD countries (OECD 2013).

The control variables for country and discipline remained statistically significant in their relationship to research specialisation. Specialisation in pure basic or pure applied research was significant and negatively associated with being located in China and Malaysia (compared to the reference category of USA), which was to be expected given the aversion towards specialisation found in the descriptive results. The other regional patterns that remained significant were the greater likelihood of pure basic researchers to be located in the Western European countries of Italy, Norway, the Netherlands and Finland. Disciplinary differences continued to reflect Becher's (1994) typologies. Pure basic researchers were significantly more likely to be located in the humanities, while pure applied researchers were significantly more likely to be located in engineering, agriculture, medicine and commerce.

In summary, pure basic researchers could be more clearly differentiated from others based on their research funding, professional norms and perceptions of institutional expectations, while pure applied researchers share more in common with those engaged in both basic and applied research.

\section{Discussion and conclusion}

Basic research is an activity that continues to define academic work at most research universities around the world. The majority of academics $(61 \%)$ in our data material based on more than 10,000 researchers from 15 countries report significant engagement in basic research, and very few report no engagement in basic research (7\%). Although these aggregate results mask considerable diversity across countries, there is little evidence that basic research is a minority preoccupation in any of the countries for which we have data. However, more academics are engaged in applied research than basic research. Those academics that engaged in both types did not do so equally, with more academics leaning towards applied over basic. This suggests there are some trade-offs between the two activities. Therefore, basic research may be considered to be an important part of most academics' research, but often secondary to applied research. Our cross-sectional data do not allow us to investigate the extent to which theoretical research has declined from a dominant position, but the resilience of theoretical research lends stronger support to the more nuanced position of the triple helix model (Etzkowitz and Leydesdorff 2000). The shift towards Mode 2 or practical research appears to be emergent, rather than complete, with universities remaining a core producer of theoretical knowledge.

Our research generally supports Stokes (1997) since a main result is that combining basic and applied work is the norm for the majority of academics, regardless of country and discipline. It also supports earlier work claiming that traditional academic orientations remain strong in universities, even in periods of decreased core funding and increased pressure for various forms of societal engagement (Ylijoki et al. 2011). We still do not have sufficient evidence to conclude whether the frequent "hybrid profiles" of academics 
are productive and meaningful or whether they to a greater extent represent incommensurable activities leading to stress and insecurity (c.f. Davies 2013).

Disciplinary-based analyses showed basic research to be relatively weaker in the professional social science disciplines (e.g. business and education) and the applied sciences (engineering, agriculture and medicine). Academics within these disciplines were rarely primarily focused on theoretical research, but these patterns probably reflect the cognitive knowledge structures of these disciplines and their orientation towards practical outputs (Becher and Trowler 2001), rather than a weakening importance of theoretical research. The diversity within these disciplines also suggests that theoretical research can be an important, and even dominant, motivation. Highly practical results may be the starting point for conducting research in some applied disciplines, but academic prestige has a strong appeal for academics across various disciplines (Hakala and Ylijoki 2001). Nevertheless, with up to around one-third of academics in the applied sciences engaging purely in applied research, compared to less than one-fifth in other fields, a complete devotion towards practice orientated research is clearly more relevant in certain fields. This lends stronger support to the field-dependent interpretations offered by Weingart (1997) and Woolf (2008) in their competing frameworks for understanding changes to the science system.

Our cross-country results are less easy to explain and should be treated with caution due to the limitations of the data. The strong position of basic research in Finnish and Norwegian universities has been noted previously (see Ylijoki et al. 2011; Gulbrandsen and Kyvik 2010), but our international results from other countries suggest a more nuanced interpretation. Although more academics were significantly engaged in basic research than applied research in Norway and the Netherlands (with an equal division in Italy), these countries were the exceptions. Applied research dominated in most countries, particularly in Australia where academics were roughly twice as likely to specialise or lean towards applied research compared to basic research. This tendency was also noticeable in the USA and Hong Kong. One possibility is that applied research engagement reflects academic governance systems, with a stronger academic oligarchy protecting the place of basic research compared to market-driven systems (Clark 1983), but this was not consistent with results in all countries.

Institutional norms emphasising research commercialisation were weakest in Mexico, Argentina and Brazil, and relatively weaker in most European countries (see Table 4 of "Appendix"). This was consistent with traditional systems of bureaucratised governance within Latin American universities, which have been criticised for creating few incentives to engage in research commercialisation and research productivity more generally (Thorn and Soo 2006). However, although each of these institutional aspects differentiated between individuals within countries in consistent ways, the differences across countries remained. For example, strong institutional-level norms towards research commercialisation in the UK were not consistent with the rather moderate engagement in applied research in this country. Nor were weak commercialisation norms in Brazil and Argentina consistent with the moderate engagement in applied research and weak engagement in basic research. In Latin America, it has been claimed that university discourse has shifted enthusiastically towards Mode 2, but the academic evaluation system remains rooted in Mode 1 outputs, leading to an "evaluation system schizophrenia" (Arocena and Sutz 2001, p. 1231). Engagement in basic and applied research clearly has strong country-level features, implying that generalised statements about basic or applied research must be qualified for local context.

China and Malaysia are particularly interesting countries because both applied research and basic research were strong self-characterisations. Neither country has strong university research traditions, but their respective governments have recently increased their expectations for basic research as part of innovation and development plans (Zhu and Gong 2008; Lee et al. 2013). Up 
until the mid-1980s, Chinese universities were predominantly teaching focused, receiving no specific funding for research. Basic research was the responsibility of the Chinese Academy of Science and other national research institutes, while applied research was conducted by research institutes in industrial sectors (Zhu and Gong 2008). Malaysian universities have also been largely teaching focused and lacked an academic research culture (Lee 2003). Strong engagement in both basic and applied research may reflect an emerging research culture which supports both basic and applied research. According to Mohrman et al. (2008), at the same time that Chinese institutions are encouraging basic research, universities are also responding to demands for applied research as a service to society. This appeared to translate into individuallevel norms. Academics in these two countries were most likely to report stronger agreement that academics had a professional obligation to apply their knowledge to problems in society.

However, the results for China and Malaysia also highlight a broader difficulty in defining basic and applied research engagement. Even though we are confident that concepts of basic/ theoretical research and applied/practically oriented research were understood in each country and the investigation teams went to great lengths to ensure translations were accurate and comparable, there is a risk that subtle differences in understanding might be responsible for parts of the differences across countries in responses. Academics in some countries may declare a greater engagement in basic research due to, for example, a broader definition of what "theoretical" or "practical" research means. This also goes beyond linguistic differences to general understanding, acceptance and reward structures for basic and applied research. The strong relationship between individual norms, institutional expectations and self-reported engagement in applied research could indicate that academics are responsive to institutional policies supporting applied research. Unfortunately, individuals may also simply exaggerate behaviours in accordance with what they perceive to be the norms of their discipline and institution. This has implications for the growing expectations of research "relevance" from higher education stakeholders around the world (Enders and Musselin 2008). If most academics genuinely believe they are already deeply engaged in practical research, it may indicate they hold a different understanding of applied and practically oriented research compared to the policy makers articulating the need for greater engagement.

We have shown that the emphasis on applied research is not only related to funding and university strategies; it is even more strongly related to individual norms about academics obligations. National and institutional policies for more applied and practically related activities are therefore not necessarily out of touch with core academic values. Our main finding is that different types of research activities are mixed and combined at the individual level, despite variations between countries and between academic disciplines. This does not imply that the balance between basic and applied research is equal, or that basic research has not lost its preeminence, but it does imply that basic research retains a core position within the research mind sets of most academics. As such, our results can be interpreted as a warning against policies striving for clear division in the higher education landscape between institutions primarily doing basic research and others applied. This seems out of touch with widely shared norms and practices among the researchers themselves, who overwhelming combine these activities.

Open Access This article is distributed under the terms of the Creative Commons Attribution License which permits any use, distribution, and reproduction in any medium, provided the original author(s) and the source are credited.

\section{Appendix}

See Tables 4, 5 and 6. 


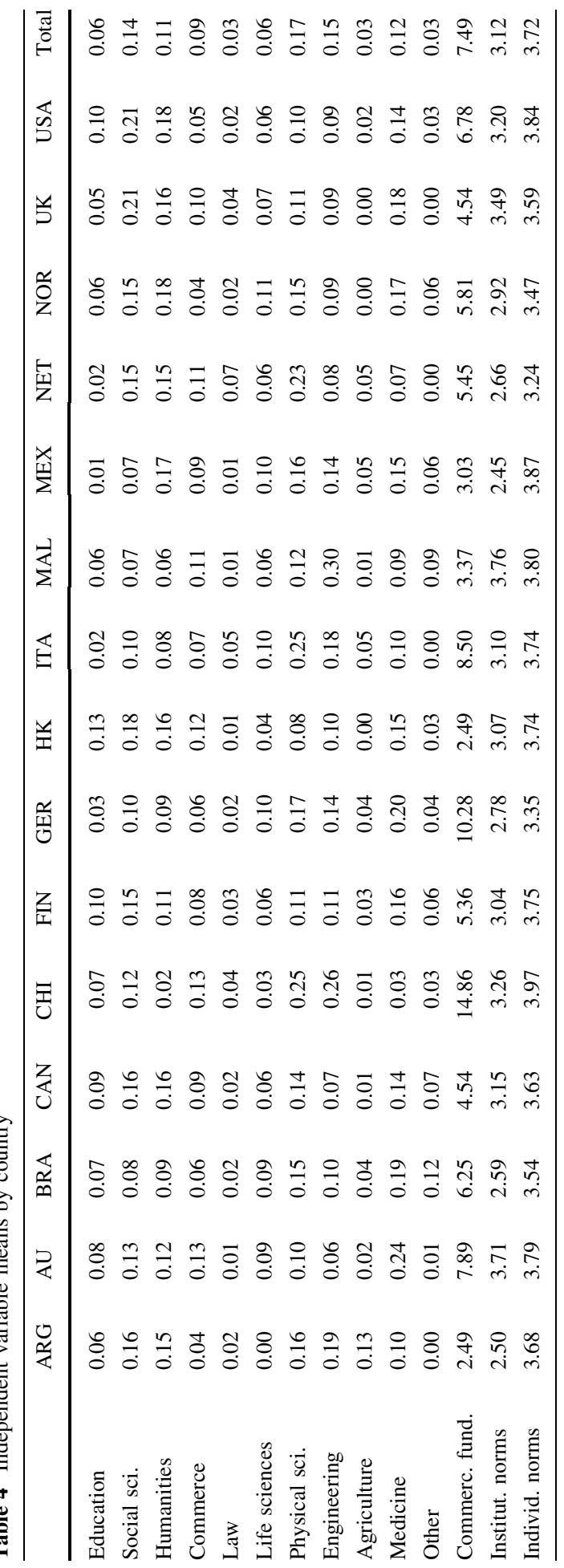


Table 5 Basic and applied research orientation classifications by country, percentages

\begin{tabular}{lrlllllr}
\hline & $\begin{array}{l}\text { Pure } \\
\text { basic }\end{array}$ & $\begin{array}{l}\text { Leaning } \\
\text { towards basic }\end{array}$ & $\begin{array}{l}\text { Equally basic and } \\
\text { applied }\end{array}$ & $\begin{array}{l}\text { Leaning towards } \\
\text { applied }\end{array}$ & $\begin{array}{l}\text { Pure } \\
\text { applied }\end{array}$ & Total & $\mathrm{n}$ \\
\hline Argentina & 16 & 17 & 18 & 26 & 23 & 100 & 691 \\
Australia & 10 & 15 & 19 & 29 & 26 & 100 & 883 \\
Brazil & 16 & 18 & 18 & 28 & 20 & 100 & 366 \\
Canada & 17 & 18 & 20 & 23 & 22 & 100 & 861 \\
China & 2 & 15 & 53 & 26 & 4 & 100 & 2,299 \\
Finland & 20 & 19 & 20 & 20 & 21 & 100 & 430 \\
Germany & 16 & 20 & 19 & 23 & 22 & 100 & 802 \\
Hong Kong & 12 & 18 & 24 & 25 & 20 & 100 & 597 \\
Italy & 25 & 20 & 12 & 19 & 24 & 100 & 1191 \\
Malaysia & 5 & 18 & 46 & 23 & 8 & 100 & 626 \\
Mexico & 15 & 21 & 21 & 26 & 16 & 100 & 477 \\
Netherlands & 25 & 19 & 23 & 16 & 17 & 100 & 410 \\
Norway & 24 & 23 & 21 & 10 & 22 & 100 & 499 \\
UK & 17 & 17 & 24 & 29 & 13 & 100 & 637 \\
USA & 13 & 18 & 19 & 28 & 23 & 100 & 648 \\
Total & 14 & 18 & 27 & 24 & 17 & 100 & 11,417 \\
\hline
\end{tabular}

Table 6 Basic and applied research orientation classifications, percentages within discipline of current academic unit

\begin{tabular}{lrlllllr}
\hline & $\begin{array}{l}\text { Pure } \\
\text { basic }\end{array}$ & $\begin{array}{l}\text { Lean. } \\
\text { towards } \\
\text { basic }\end{array}$ & $\begin{array}{l}\text { Equally } \\
\text { basic and } \\
\text { applied }\end{array}$ & $\begin{array}{l}\text { Lean. } \\
\text { towards } \\
\text { applied }\end{array}$ & $\begin{array}{l}\text { Pure } \\
\text { applied }\end{array}$ & Total & $n$ \\
\hline Teacher training and education & 7 & 12 & 32 & 29 & 19 & 100 & 677 \\
Humanities & 28 & 21 & 26 & 16 & 9 & 100 & 1,347 \\
Social/behavioural sciences & 19 & 19 & 24 & 23 & 16 & 100 & 1,127 \\
Business and economics & 8 & 15 & 31 & 28 & 18 & 100 & 980 \\
Law & 18 & 20 & 34 & 18 & 10 & 100 & 301 \\
Life sciences & 22 & 26 & 23 & 17 & 12 & 100 & 694 \\
Physical sciences, mathematics, & 18 & 24 & 29 & 19 & 10 & 100 & 1,809 \\
$\quad$ computer sciences & 3 & 13 & 32 & 32 & 21 & 100 & 1,667 \\
Engineering, architecture & 4 & 8 & 23 & 31 & 35 & 100 & 305 \\
Agriculture & 9 & 16 & 18 & 27 & 30 & 100 & 1,322 \\
Medical and health sciences & 8 & 15 & 33 & 24 & 20 & 100 & 334 \\
Other & 14 & 18 & 27 & 24 & 17 & 100 & 10,563 \\
Total & & & & & & & \\
\hline
\end{tabular}

\section{References}

Albert, M. (2003). Universities and the market economy: The differential impact on knowledge production in sociology and economics. Higher Education, 45(2), 147-182. 
Altbach, P. G. (2001). Academic freedom: International realities and challenges. Higher Education, 41(1-2), $205-219$.

Arocena, R., \& Sutz, J. (2001). Changing knowledge production and Latin American universities. Research Policy, 30, 1221-1234.

Auranen, O., \& Nieminen, M. (2010). University research funding and publication performance-An international comparison. Research Policy, 39(6), 822-834. doi:10.1016/j.respol.2010.03.003.

Becher, T. (1994). The significance of disciplinary differences. Studies in Higher Education, 19(2), $151-161$.

Becher, T., \& Trowler, P. (2001). Academic tribes and territories: Intellectual enquiry and the culture of disciplines. Philadelphia, PA: Open University Press.

Calvert, J. (2006). What's special about basic research. Science, Technology and Human Values, 31, 199-220.

Clark, B. R. (1983). The higher education system. Berkeley: University of California Press.

Clark, B. R. (1998). The entrepreneurial university: Demand and response. Tertiary Education and Management, 4(1), 5-16.

Cummings, W. K., \& Finkelstein, M. J. (2012). 2. Concepts and methods. In W. K. Cummings \& M. J. Finkelstein (Eds.), Scholars in the changing American Academy: New contexts, new rules, and new roles (pp. 15-26). Dordrecht: Springer.

Davies, S. (2013). Research staff and public engagement: A UK study. Higher Education, 66(6), 725-739. doi:10.1007/s10734-013-9631-y.

Enders, J. (2013). The university in the audit society: On accountability, trust and markets. In L. Engwall \& P. Scott (Eds.), Trust in universities (pp. 53-62). London: Portland Press.

Enders, J., \& Musselin, C. (2008). Back to the future? The academic professions in the 21st century. In Higher education to 2030: Demography (Vol. 1, pp. 125-150, Demography). Paris: OECD.

Etzkowitz, H. (1998). The norms of entrepreneurial science: Cognitive effects of the new universityindustry linkages. Research Policy, 27(8), 823-833. doi:10.1016/S0048-7333(98)00093-6.

Etzkowitz, H., \& Leydesdorff, L. (2000). The dynamics of innovation: From National Systems and "Mode 2" to a Triple Helix of university-industry-government relations. Research Policy, 29(2), 109-123.

Gibbons, M., Limoges, C., Nowotny, H., Schwartzman, S., Scott, P., \& Trow, M. (1994). The new production of knowledge: The dynamics of science and research in contemporary societies. London: Sage.

Godin, B. (1998). Writing performative history: the new New Atlantis? Social Studies of Science, 28(3), 465-483.

Gulbrandsen, M., \& Kyvik, S. (2010). Are the concepts basic research, applied research and experimental development still useful? An empirical investigation among Norwegian academics. Science and Public Policy, 37(5), 343-353.

Gulbrandsen, M., \& Langfeldt, L. (2004). In search of Mode 2: The nature of knowledge production in Norway. Minerva, 42, 237-250.

Hakala, J., \& Ylijoki, O.-H. (2001). Research for whom? Research orientations in three academic cultures. Organization, 8(2), 373-380. doi:10.1177/1350508401082021.

Hessels, L. K., \& Van Lente, H. (2008). Re-thinking new knowledge production: A literature review and a research agenda. Research Policy, 37(4), 740-760.

Irvine, J., \& Martin, B. R. (1984). Foresight in science: Picking the winners. London: Pinter.

Kyvik, S. (2007). Changes in funding of university research. Consequences for problem choice and research output of academic staff. In J. Enders \& B. Jongbloed (Eds.), Public-private dynamics in higher education: Expectations, developments and outcomes (pp. 387-411). Bielefeld: Transcript Verlag.

Lee, M. N. N. (2003). The academic profession in Malaysia and Singapore: Between bureaucratic and corporate cultures. In P. G. Altbach (Ed.), The decline of the guru: The academic profession in the third world (pp. 135-166). Chestnut Hill: Boston College.

Lee, M. N. N., Sirat, M., Da, W. C., \& Karpudewan, M. (2013). Appendix C: Effectiveness of research and innovation management at policy and institutional levels in Malaysia. In A. Olsson \& L. Meek (Eds.), Effectiveness of Research and Innovation Management at Policy and Institutional Levels (pp. 90-120). Paris: OECD.

Mohrman, K., Ma, W., \& Baker, D. (2008). The research university in transition: The emerging global model. Higher Education Policy, 21(1), 5-27.

Mowery, D., \& Sampat, B. (2005). The Oxford Handbook of Innovation. In J. Fagerberg, D. C. Mowery, \& R. R. Nelson (Eds.), Universities in national innovation systems (pp. 209-239). Oxford: Oxford University Press.

Musselin, C. (2005). European academic labor markets in transition. Higher Education, 49(1-2), $135-154$.

Nowotny, H., Scott, P., \& Gibbons, M. (2003). Introduction: 'Mode 2' revisited: The new production of knowledge. Minerva, 41(3), 179-194. doi:10.1023/a:1025505528250. 
OECD. (2002). Frascati manual 2002: Proposed standard practice for surveys on research and experimental development. Paris: OECD.

OECD. (2013). MSTI main science and technology indicators. Paris: OECD.

RIHE (Ed.). (2008). The changing academic profession in international comparative and quantitative perspectives (Vol. 12, RIHE International Seminar Reports). Hiroshima: Research Institute for Higher Education, Hiroshima University.

Rip, A. (2004). Strategic research, post-modern universities and research training. Higher Education Policy, $17(2), 153-166$.

Shinn, T. (2002). The triple helix and new production of knowledge prepackaged thinking on science and technology. Social Studies of Science, 32(4), 599-614.

Slaughter, S., \& Leslie, L. L. (1997). Academic capitalism: Politics, policies, and the entrepreneurial university. Baltimore: The Johns Hopkins University Press.

Stokes, D. E. (1997). Pasteur's quadrant: Basic science and technological innovation. Washington, DC: Brookings Institution Press.

Stuart, T. E., \& Ding, W. W. (2006). When do scientists become entrepreneurs? The social structural antecedents of commercial activity in the academic life sciences. American Journal of Sociology, $112(1), 97-144$.

Thorn, K., \& Soo, M. (2006). Latin American universities and the third mission: Trends, challenges, and policy options [Working Paper, no. 4002]. World Bank Policy Research. The World Bank.

Vabø, A., \& Ramberg, I. (2009). Arbeidsvilkår i norsk forskning. Oslo: NIFU STEP.

van Rijnsoever, F. J., \& Hessels, L. K. (2011). Factors associated with disciplinary and interdisciplinary research collaboration. Research Policy, 40(3), 463-472. doi:10.1016/j.respol.2010.11.001.

Weingart, P. (1997). From "Finalization" to "Mode 2": Old wine in new bottles? Social Science Information, 36(4), 591-613.

Woolf, S. H. (2008). The meaning of translational research and why it matters. JAMA, 299(2), 211-213.

Ylijoki, O.-H., Lyytinen, A., \& Marttila, L. (2011). Different research markets: A disciplinary perspective. Higher Education, 62(6), 721-740. doi:10.1007/s10734-011-9414-2.

Zhu, Z., \& Gong, X. (2008). Basic research: Its impact on China's future. Technology in Society, 30(3-4), 293-298. doi:10.1016/j.techsoc.2008.05.001. 